\title{
Topological T-duality and T-folds
}

\author{
Peter Bouwknegt ${ }^{1,2}$ and Ashwin S. Pande ${ }^{1}$
}

${ }^{1}$ Department of Theoretical Physics, Research School of Physical Sciences and Engineering, The Australian National University, Canberra, ACT 0200, Australia

peter.bouwknegt@anu.edu.au; asp105@rsphysse.anu.edu.au ${ }^{2}$ Department of Mathematics, Mathematical Sciences Institute, The Australian National University, Canberra, ACT 0200, Australia

\begin{abstract}
We explicitly construct the $C^{*}$-algebras arising in the formalism of Topological T-duality due to Mathai and Rosenberg from string-theoretic data in several key examples. We construct a continuous-trace algebra with an action of $\mathbb{R}^{d}$ unique up to exterior equivalence from the data of a smooth $\mathbb{T}^{d}$-equivariant gerbe on a trivial bundle $X=W \times \mathbb{T}^{d}$. We argue that the "non-commutative T-duals" of Mathai and Rosenberg [7] should be identified with the non-geometric backgrounds well known in string theory. We also argue that the $C^{*}$-algebra $\mathcal{A} \rtimes_{\left.\alpha\right|_{\mathbb{Z}^{d}}} \mathbb{Z}^{d}$ should be identified with the T-folds of Hull [1] and Belov et al. [2] which geometrize these backgrounds.

We identify the charge group of D-branes on T-fold backgrounds in the $C^{*}$-algebraic formalism of Topological T-duality. We also study D-branes on T-fold backgrounds. We show that the $K$-theory bundles of [13] give a natural description of these objects.
\end{abstract}

e-print archive: http://lanl.arXiv.org/abs/hep-th//0810.4374 


\section{Introduction}

It has been known for a some time that string theories may be defined on backgrounds which are not conventional geometries (see $[1-4,9]$ and references therein). We briefly review the construction of such backgrounds here following [1]. Some of these non-geometric backgrounds may be constructed by considering "parametrized families" of string theories with the target space of each string theory being a familiar space ${ }^{1}$ such as $\mathbb{T}^{d}$ or the ALE spaces.

String theories with target a $d$-dimensional torus possess a "large" symmetry group (isomorphic to $\mathrm{O}(d, d, \mathbb{Z})$ ) of which the "geometric" subgroup $\mathrm{GL}(d, \mathbb{Z}) \subset \mathrm{O}(d, d, \mathbb{Z})$ is generated by large diffeomorphisms of $\mathbb{T}^{d}$ while the rest are generated by T-dualities combined with shifts of the Kalb-Ramond field.

Now consider families of string theories parametrized by a space $W$, i.e. for each point of $W$ we consider a string theory with target $\mathbb{T}^{d}$. It is clear that after moving around any topologically non-trivial loop in $W$, the theory must return to itself up to a symmetry, i.e., we have a monodromy. The continuous part of the symmetry may be gauged away and hence the monodromy will take values in the large symmetry group of the theory on the $\mathbb{T}^{d}$ fiber. If every monodromy lies in the geometric subgroup of the symmetry group then the parametrized family of theories is actually equivalent to a certain limit of string theory with target a $\mathbb{T}^{d}$-bundle over $W$ with structure group the geometric group.

If the monodromy does not lie in the geometric subgroup; however, we cannot view the parametrized family as being defined by a theory on a geometric target spacetime. In this case it has been argued that the target is (roughly speaking) a $\mathbb{T}^{d}$ fibration over $W$ in which the fibers are "glued together using T-dualities". Such a space is not a geometry in the ordinary sense of the term and has been termed a "T-fold" $[1,2]$ or a "monodrofold" $[3,4]$.

Some T-folds arise as T-duals of ordinary geometries, and hence string theory is certainly well defined on them, even as a quantum theory. Conversely, Hull has shown that string theory on a general T-fold background may be defined as a theory with a sigma-model-like action with target a geometric background together with a set of constraints. He has argued

\footnotetext{
${ }^{1}$ We only consider $\mathbb{T}^{d}$ as a target space here.
} 
that this action may be quantized to obtain the full quantum theory associated with the T-fold [1]. This background is a (possibly non-principal) $\mathbb{T}^{2 d}$ fibration over $W$ referred to in [2] as the "correspondence space".

We give a brief outline of the basic theory of T-folds here closely following [11], Section 2. We consider a $\mathbb{T}^{2 d}$ fibration over $W$. The formalism of $[1,11]$ introduces a new indefinite metric field $L^{I J}(Y)$ on this fibration. The physical spacetime is defined by choosing for each $y \in Y$, a $\mathbb{T}^{d} \subseteq \mathbb{T}^{2 d}$ which is null with respect to $L$. This defines a $\mathbb{T}^{d}$ fibration over $W$. To be precise ${ }^{2}$ we introduce projectors $\Pi_{I}^{i}, \tilde{\Pi}_{i I} i=1, \ldots, n$, such that the matrix

$$
\Pi=\left(\begin{array}{c}
\Pi_{I}^{i} \\
\tilde{\Pi}_{i I}
\end{array}\right), \quad i=1, \ldots, n
$$

defines a choice of coordinates on each $\mathbb{T}^{2 d}$ fiber in which $L$ has the form

$$
\left(\begin{array}{ll}
0 & 1 \\
1 & 0
\end{array}\right)
$$

then, $L^{I J} \Pi_{I}^{i} \Pi_{J}^{j}=L^{I J} \tilde{\Pi}_{i I} \tilde{\Pi}_{j J}=0$. Let $\left(\sigma^{0}, \sigma^{1}\right)$ be the coordinates on the worldsheet, $\mathbb{X}^{I}, I=1, \ldots, 2 d$, be coordinates on the $\mathbb{T}^{2 d}$ fiber and $Y^{A}, A=$ $1, \ldots, n$, coordinates on the base $W$ of the fibration. If we let $X=\Pi \mathbb{X}$ and $\tilde{X}=\tilde{\Pi} \mathbb{X}$ then $X, Y$ are the coordinates on the physical spacetime and $\tilde{X}, Y$ are the coordinates on the T-dual spacetime.

The T-fold action is then

$$
\mathcal{L}=-\frac{1}{2} \mathcal{H}_{I J}(Y) \eta^{\alpha \beta} \partial_{\alpha} \mathbb{X}^{I} \partial_{\beta} \mathbb{X}^{J}-\eta^{\alpha \beta} \mathcal{I}_{I A}(Y) \partial_{\alpha} \mathbb{X}^{I} \partial_{\beta} Y^{A}+\mathcal{L}_{N}(Y)
$$

Here $\mathbb{X}^{I}$ and $Y^{A}$ are worldsheet fields taking values in the base and fiber of the fibration over $W$. Also, $\mathcal{H}$ is a family of metrics on the $\mathbb{T}^{2 d}$ fiber parametrized by the base $W$, and $\mathcal{I}$ is a "connection" for the $\mathbb{T}^{2 d}$ fibration over $W$ (see $[1,11]$ for details). Also, $\mathcal{L}_{N}$ is the action for the fields with target the base $W$. We have the explicit form

$$
\mathcal{H}=\left(\begin{array}{cc}
\mathcal{H}_{i j} & \mathcal{H}_{i}{ }^{j} \\
\mathcal{H}^{i}{ }_{j} & \mathcal{H}^{i j}
\end{array}\right)=\left(\begin{array}{cc}
G_{i j}-B_{i k} G^{k l} B_{l j} & B_{i k} G^{k j} \\
-G^{i k} B_{k j} & G^{i j}
\end{array}\right),
$$

where $G$ is the metric on the $\mathbb{T}^{2 d}$ fibers and $B$ is the restriction of the $B$ field to the $\mathbb{T}^{2 d}$ fibers (that is, we only consider the components of the metric and the $B$ field along the $\mathbb{X}$ coordinates).

\footnotetext{
${ }^{2}$ See [11, Section 2.1].
} 
The metric $G$ and $B$ field on the physical spacetime may be obtained from the above data (equation (4.9) of [1]) as

$$
\begin{aligned}
G^{i j} & =\Pi^{i I} \Pi^{j J} \mathcal{H}_{I J}, \\
B_{i j} G^{j k} & =\tilde{\Pi}_{i}^{I} \Pi^{k J} \mathcal{H}_{I J} .
\end{aligned}
$$

Note that these are exactly the entries in the second column of the matrix (equation (1.2) above) for $\mathcal{H}$. It is clear that if we interchange $\Pi$ and $\tilde{\Pi}$, we will obtain the first column of $\mathcal{H}$ above, which is exactly the T-dual metric and $B$ field and would be associated to the T-dual spacetime.

Thus the data of a T-fold (namely the metric $\mathcal{H}$ and the fixed choice of projections $\Pi, \tilde{\Pi})$ determine two $\mathbb{T}^{d}$ fibrations over $W$, each with their own metric and $B$ field, one corresponding to the physical spacetime and the other to one of its T-duals. Exactly which T-dual is chosen is a matter of convention and decided by the form of the metric $L$. In the conventions of [11], the T-fold is constructed from the original spacetime and the one obtained by T-dualizing along all of the $\mathbb{T}$-orbits simultaneously. When both the original spacetime and the T-dual exist as geometric backgrounds, the T-fold is the fiber product of these two spacetimes, it is the "correspondence space" (see below). On it, there is only the tensor field $\mathcal{H}$ which, as discussed above, contains the original metric and $B$ field. However, T-folds continue to exist as geometric spaces even when the original space or its full T-dual is non-geometric. An explicit example of this is the full T-dual $\mathbb{T}^{3}$ viewed as a trivial $\mathbb{T}^{2}$-bundle over $\mathbb{T}$ with $H$-flux which we will discuss in detail in Section 4 below.

Now non-geometric backgrounds have also been seen in the $C^{*}$-algebraic formalism of Topological T-duality of [7]. In this paper we study the relationship of these two formalisms to each other. (See [9] for another viewpoint on this issue.) In what follows, we use the term "T-fold" to refer to the geometrization of the non-geometric T-dual, i.e., to the (possibly nonprincipal) $\mathbb{T}^{2 d}$ fibration over $W$ referred to as the "correspondence space" in [2]. This is the same as the correspondence space of [6-8] (see below).

We now outline the two formalisms that we will study in the following sections: In the formalism of Topological T-duality of [7], we begin with a $C^{*}$-dynamical system $\left(\mathcal{A}, \alpha, \mathbb{R}^{d}\right)$ such that the spectrum of $\mathcal{A}$ denoted by $\hat{\mathcal{A}}$ is a principal $\mathbb{T}^{d}$-bundle $p: X \rightarrow W$ and the $\mathbb{R}^{d}$-action on $\mathcal{A}$ induces the given $\mathbb{T}^{d}$-action on $X=\hat{\mathcal{A}}$. Here $\hat{\mathcal{A}}$ is supposed to be a model for the topological type of a target space with a $\mathbb{T}^{d}$-isometry. The cohomology class of the $H$-flux on the target space is modelled by the Dixmier-Douady 
class of $\mathcal{A}$. The topological T-dual of $\mathcal{A}$ is [7] the $C^{*}$-dynamical system $\left(\mathcal{A} \rtimes_{\alpha} \hat{\mathbb{R}}^{d}, \hat{\alpha}, \hat{\mathbb{R}}^{d}\right)$. To each such $C^{*}$-dynamical system, there is associated a function $f: W \rightarrow \mathbb{T}^{d(d-1) / 2}$ termed the Mackey obstruction map ${ }^{3}$ of the system. When this map is nullhomotopic (we say "there are no Mackey obstructions") the spectrum $X^{\#}$ of this $C^{*}$-algebra is again a principal circle bundle $q: X^{\#} \rightarrow W$ termed the T-dual bundle. The authors of [7] also show that in this case there is a "correspondence space" homeomorphic to the fibered product $X \times_{W} X^{\#}$ (the spectrum of $\mathcal{A} \rtimes_{\left.\alpha\right|_{\mathbb{Z}^{d}}} \mathbb{Z}^{d}$ ) such that the following diagram of spaces commutes:

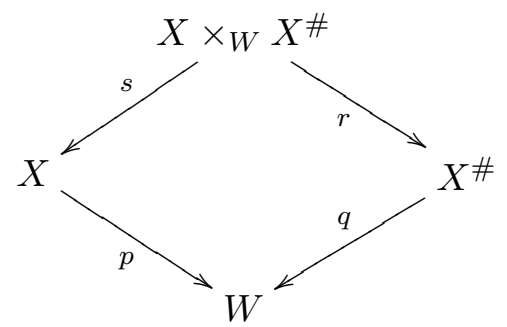

Here, $X, X^{\#}$ and $X \times_{W} X^{\#}$ are principal $\mathbb{T}^{d}$-bundles over $W$ with specified integral three-cohomology classes representing the $H$-flux, and all the maps are bundle projections. In addition, the pullbacks of the $H$-fluxes along $r, s$ agree. If there are Mackey obstructions, however, the T-dual is in general a non-commutative space and the above diagram of spaces does not exist. However, there is a similar diagram of $C^{*}$-algebras with the correspondence space replaced by the $C^{*}$-algebra $\mathcal{A} \rtimes_{\left.\alpha\right|_{\mathbb{Z}^{d}}} \mathbb{Z}^{d}$. We will argue in Section 2 below that this algebra is a natural analog of the T-folds of Hull when the $\mathrm{T}$-dual is nongeometric.

The formalism of [2] considers a smooth principal $\mathbb{T}^{d}$-bundle $p: X \rightarrow W$ (representing the target space) together with a smooth $\mathbb{T}^{d}$-equivariant gerbe with connection on $X$ (this is the conventional model for the $H$-flux). The $\mathbb{T}^{d}$-equivariance is needed in order to satisfy the conditions for gauging the sigma model on the target space (see $[1,2])$. The authors define a certain obstruction cocycle $m_{\alpha \beta}^{I J}$ which defines a class in $H^{1}\left(W, \mathbb{Z}^{m}\right), m=d(d-1) / 2$ with $I, J=1, \ldots, d$, such that the T-dual is geometric if and only if this class is zero. In this case, as shown in [2], we may define a smooth correspondence space, which gives a commutative diamond of spaces exactly as in equation (1.4). If some $m_{\alpha \beta}^{I J}$ define non-zero cohomology classes in $H^{1}\left(W, \mathbb{Z}^{m}\right)$, a geometric T-dual does not exist. In this case, the correspondence space of [2] is a geometric space but it is a non-principal $\mathbb{T}^{2 d}$-bundle over $W$.

\footnotetext{
${ }^{3}$ See Section 2 below.
} 
If we denote this correspondence space by $C$ here, we have a "diagram"

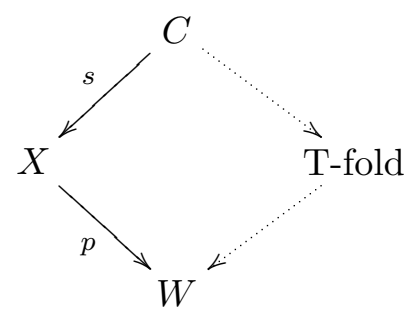

Here the dotted arrows are purely indicative, no quotient is implied.

Now the formalism of [2] is defined in the category of smooth manifolds, whereas the formalism of [7] is in a category of $C^{*}$-algebras. A connection between these two seemingly different formalisms is of interest. Note that we do obtain the same correspondence space in both formalisms when, in one case, the Mackey obstruction vanishes and in the other, when all the $m^{I J}$ are zero. It is natural to conjecture that the two obstructions are related. We will show below that this is indeed the case. We will also construct the continuous-trace algebra associated with certain types of $H$-flux on trivial torus bundles over a base $W$ directly from the string theoretic data of [2]. In particular, we argue that the "non-commutative T-duals" discussed in [7] should then be viewed as topological approximations to the T-folds of Hull. We relate D-branes on the correspondence space to the formalism of Topological T-duality in Section 3 below. We also compare our findings to the string-theoretic calculations in [11].

\section{A Topological approximation of $\boldsymbol{H}$-flux}

In this section, we compare the formalisms of Mathai and Rosenberg $[6,7]$ to the string theoretic T-dual studied by Belov et al. [2]. Note that the $C^{*}$-algebraic formalism is only concerned with the topological aspects of T-duality, and hence a certain loss of information is to be expected on passing to it from string theory.

We will see that the reason why the $C^{*}$-algebraic formulation of Topological T-duality agrees with string theory is due to the structure of the $H$-flux in string theory. It is well known that the $H$-flux in string theory is required to satisfy the WZW condition namely

$$
\mathcal{L}_{t} H=0,
$$


where $t$ is a tangent vector field on $X$ which is associated to the torus action on $X$. As shown in $[1,2,10]$ this forces the $H$-flux to have the structure

$H=\frac{1}{6} p^{*} H_{0}^{I J K} \wedge \Theta_{I} \wedge \Theta_{J} \wedge \Theta_{K}+\frac{1}{2} p^{*} H_{1}^{I J} \wedge \Theta_{I} \wedge \Theta_{J}+p^{*} H_{2}^{I} \wedge \Theta_{I}+p^{*} H_{3}$,

where $\Theta_{I}, I=1, \ldots d$, are the components of the connection form on $X$ and $H_{k} \in \Omega^{k}(W)$ (we use the notation of [1] here, see equations (1.1), and (1.2) of that paper). We will see below that in several interesting cases this structure enables us to construct the $C^{*}$-algebraic T-dual.

In [1], the authors consider a smooth $\mathbb{T}^{d}$-equivariant gerbe with connection on $X$. In the case when the T-dual is non-geometric, they show that the gerbe connection naturally gives a $\mathbb{T}^{2 d}$-bundle over $W$ with structure group $\mathrm{GL}(2 d, \mathbb{Z})$. They identify this with the correspondence space.

Hull [1, Theorem 2.2] obtains an integral one-cocycle $m_{\alpha \beta} \in H^{1}\left(W, \wedge^{2} \mathbb{Z}^{d}\right)$ from a gerbe connection on $X$. We will show below that the homotopy class of the Mackey obstruction map $f: W \rightarrow \mathbb{T}^{d(d-1) / 2}$ of $[7,13]$ can be identified with the cohomology class of this cocycle.

As noted in [1], the class $m_{\alpha \beta}$ vanishes iff the T-dual is geometric and then the correspondence space is the fibered product of the original space and the T-dual. In the $C^{*}$-algebraic formalism, the spectrum of $\mathcal{T}$ is exactly this fiber product when the Mackey obstruction vanishes. Hence it seems natural to conjecture that $m_{\alpha \beta}$ is related to the Mackey obstruction.

Below, we will explicitly construct the Mackey obstruction map from the data of a smooth equivariant gerbe and show that its homotopy class is exactly $m_{\alpha \beta}$. A heuristic argument for identifying the two is as follows: In [1], it is shown that the component of the $H$-flux with two "legs" along the torus fiber and one leg along the base determines classes ${ }^{4} m_{\alpha \beta}^{I J} \in H^{1}(W, \mathbb{Z})$. Since $m^{I J}$ is skew-symmetric in $I, J$ there will be as many such classes as the number of elements in the basis of $\wedge^{2} \mathbb{Z}^{d}$ determined by $I$. These give an element of $H^{1}\left(W, \wedge^{2} \mathbb{Z}^{d}\right)$.

In particular if $a^{I J}$ is a vector-valued differential form representing $m_{\alpha \beta}$, the component of the $H$-flux associated to $m_{\alpha \beta}$ is $a^{I J} \wedge \Theta_{I} \wedge \Theta_{J}$, where $\Theta_{I}$ is the connection form on $X$. If we could construct a continuous-trace algebra $\mathcal{A}$ having Dixmier-Douady invariant $[H]$ together with an $\mathbb{R}^{d}$-action $\alpha$ covering the $\mathbb{T}^{d}$-action on $X$ thus determining a $C^{*}$-dynamical system $[\mathcal{A}, \alpha]$ on $X$, then we would expect, ${ }^{5} m_{\alpha \beta}$ to be the cocycle representing

\footnotetext{
${ }^{4}$ Hull [1] after equation (2.20).

${ }^{5}$ In the notation of [7, Theorem 2.3].
} 
$p_{!}(H)$, i.e., $m_{\alpha \beta}=p_{!} \circ F([\mathcal{A}, \alpha])$. However, by the commutativity of the diagram in Theorem 2.3 of that paper, $p_{!} \circ F=h \circ M$ and hence $m_{\alpha \beta}$ would be the cohomology class representing the Mackey obstruction as well.

We now show that we can obtain the Mackey obstruction map itself from the string-theory calculation. We fix a basepoint $w_{0}$ in $W$ and the basepoint $(1, \ldots, 1)$ in $\mathbb{T}^{d(d-1) / 2}$.

Theorem 2.1. Given a $\mathbb{T}^{d}$-equivariant gerbe with connection on a smooth principal $\mathbb{T}^{d}$-bundle $p: X \rightarrow W$

(1) If we fix the gauge of the gerbe connection on $X$, there exists a smooth map $f: W \rightarrow \mathbb{T}^{d(d-1) / 2}$ constructed from the gerbe connection. The map is natural under pullback of gerbes.

(2) Under a gauge transformation of the gerbe connection, this map is multiplied by a phase $\gamma$, i.e., $f \rightarrow e^{i \gamma} f$.

(3) This defines a unique based map $\left(W, w_{0}\right) \rightarrow\left(\mathbb{T}^{d(d-1) / 2},(1, \ldots, 1)\right)$ such that the homotopy class of $f$ in $\left[W, \mathbb{T}^{d(d-1) / 2}\right]$ is $p_{!}([H])$. There is a geometric T-dual if and only if $f$ is nullhomotopic.

Proof. We use the notation of [2] throughout this proof. We implicitly assume the results stated therein. Let $U_{\alpha}$ be a good cover of $W$. Let $X_{\alpha}=U_{\alpha} \times \mathbb{T}^{d}$ be a cover of $X$.

(1) From [2, equation (2.4)], we see that the curvature form of the gerbe connection on $X$ is

$$
H=\frac{1}{6} p^{*} H_{0}^{I J K} \Theta_{I} \wedge \Theta_{J} \wedge \Theta_{K}+\frac{1}{2} p^{*} H_{1}^{I J} \Theta_{I} \wedge \Theta_{J}+p^{*} H_{2}^{I} \Theta_{I}+p^{*} H_{3},
$$

where $H_{k}$ are $k$-forms on $W$ and $\Theta_{I}, I=1, \ldots, d$, are the coordinates on the $\mathbb{T}^{d}$ fiber. Note that the $H$-flux is a fixed three-form and as such does not change under gauge transformations. Hence, neither do the $H_{k}$. We take $H_{0}=0$ as we are working in the context of the paper of Belov et al. [2]. This implies that $H_{1}$ is a closed form on $W$. In each patch $X_{\alpha}$, the gerbe connection $B_{\alpha}$ (whose curvature is $H$ above) may be written as

$$
B_{\alpha}=B_{2 \alpha}+B_{1 \alpha}^{I} \wedge \Theta_{I}+\frac{1}{2} B_{0 \alpha}^{I J} \wedge \Theta_{I} \wedge \Theta_{J},
$$

where $B_{k \alpha}$ are $k$-forms on $X_{\alpha}$. The equivariance condition that is required for the sigma model to be well-defined ${ }^{6} \mathcal{L}\left(\frac{\partial}{\partial \theta_{I}}\right) B_{\alpha}=0$ implies

\footnotetext{
${ }^{6}$ See $[2]$.
} 
that $\mathcal{L}\left(\frac{\partial}{\partial \theta_{I}}\right) B_{k \alpha}=0, k=0, \ldots, 3$. Hence, $\quad \mathcal{L}\left(\frac{\partial}{\partial \theta_{I}}\right) B_{0 \alpha}=0$ and so $\partial B_{0 \alpha} / \partial \theta_{I}=0$. Hence, $B_{0 \alpha}$ only depends on the coordinates of $W$ and not on the $\theta_{I}$. Thus, $B_{0 \alpha}$ is a well-defined function on $U_{\alpha} \subseteq W$.

We also have $B_{0 \alpha}^{I J}-B_{0 \beta}^{I J}=m_{\alpha \beta}^{I J}$, (see Theorem 2.2 of [2]), that is, $m_{\alpha \beta}$ is an obstruction to $B_{0 \alpha}$ being a global function on $W$. Note that $m_{\alpha \beta}^{I J}$ is the cocyle representing the Mackey obstruction as discussed above. Define $f^{I J}=\exp \left(2 \pi \mathrm{i} B_{0 \alpha}^{I J}\right), 0 \leq I<J \leq d$; it is clear that this construction is natural since, under pullback of gerbes, by definition, $B$ will map naturally. The map $f$ is smooth since $B_{0 \alpha}^{I J}$ is smooth by definition.

(2) Note that from the relation $B_{0 \alpha}^{I J}-B_{0 \beta}^{I J}=m_{\alpha \beta}^{I J}$, one might think that it would be possible to add a constant to each of the $B_{0 \alpha}^{I J}$ without affecting $H$.

As we now show this procedure is a gerby gauge transformation of $B$. Given a gerbe on $X$ the gauge transformations are generated by a global line bundle $q: K \rightarrow X$ with a connection $\nabla$ such that under a gauge transformation $B_{\alpha} \rightarrow B_{\alpha}+\Omega_{\alpha}$ where $\Omega_{\alpha}$ is the restriction of the curvature form $\Omega$ of $\nabla$ to $X_{\alpha}$. Now, $\Omega_{\alpha}$ may be written as

$$
\Omega_{\alpha}=\frac{1}{2} \Omega_{0 \alpha}^{I J} \Theta_{I} \wedge \Theta_{J}+\Omega_{1 \alpha}^{I} \Theta_{I}+\Omega_{2 \alpha}
$$

where $d \Omega_{\alpha}=0$ since $\Omega$ is the curvature of a line bundle. This implies that $d \Omega_{0 \alpha}^{I J}=0$. Hence the $\Omega_{0 \alpha}^{I J}$ are the restriction of a constant realvalued function on $X$ to $X_{\alpha}$. Let the value of this function be $\gamma$. Then, $f^{I J} \rightarrow f^{I J} \exp (\mathrm{i} \gamma)$ under a gerby gauge transformation.

(3) We choose the basepoint ${ }^{7}(1, \ldots, 1)$ in $\mathbb{T}^{d(d-1) / 2}$ and require that the value of $f$ at $w_{0}$ should be the identity $(1, \ldots, 1)$ of the Lie group $\mathbb{T}^{d(d-1) / 2}$. This fixes $f$ uniquely.

For $\mathrm{G}$ a group, let $\underline{\mathrm{G}}$ be the sheaf of $\mathrm{G}$-valued functions on $W$. Then we have a short exact sequence of sheaves $0 \rightarrow \underline{\mathbb{Z}^{m}} \rightarrow \underline{\mathbb{R}^{m}} \rightarrow \underline{\mathbb{T}^{m}} \rightarrow 0$. The first few terms in the associated long exact sequence in sheaf cohomology of $W$ are

$$
C\left(W, \mathbb{Z}^{m}\right) \rightarrow C\left(W, \mathbb{R}^{m}\right) \rightarrow C\left(W, \mathbb{T}^{m}\right) \stackrel{\beta}{\rightarrow} H^{1}\left(W, \mathbb{Z}^{m}\right),
$$

where $\beta$ is the connecting map. In the special case $m=1$, and $W=$ $S^{1}, \beta$ is the map which sends a function to its winding number and hence may be identified with the map $h$ of [7]. If we set $m=d(d-$ $1) / 2$ we see that $f^{I J}$ defines an element of $C\left(W, \mathbb{T}^{m}\right)$ and its image under $\beta$ is precisely $m_{\alpha \beta}^{I J}$. In particular, $\beta$ is the map that sends a function with values in $\mathbb{T}^{d(d-1) / 2}$ to the set of homotopy classes

\footnotetext{
${ }^{7}$ This is not unusual, for example, in [13, Lemmas 6.5 and 6.6] the Mackey obstruction map is also required to be based.
} 
$\left[W \rightarrow \mathbb{T}^{d(d-1) / 2}\right] \cong H^{1}\left(W, \mathbb{Z}^{m}\right)$. Thus the homotopy class of $f$ is $m_{\alpha \beta}$ which is the cohomology class of $H_{1}=p_{!}(H)$. If $f^{I J}$ is nullhomotopic, then $m_{\alpha \beta}$ is a coboundary and by Corollary 2.1 of [2] we see that the T-dual is a principal torus bundle. The converse is also true by Corollary 2.3 of that reference.

Thus, we see from the above that the map $f$ has exactly the behavior that the Mackey obstruction map should have (see the proof of Theorem 3.1 of [7]). In general the Mackey obstruction is only a continuous map. However, note that the above construction will always yield a smooth map. Thus, we suspect that the above construction is not reversible, there is a loss of information when passing from string theory to the formalism of Topological T-duality.

If we could naturally associate a continuous-trace algebra $\mathcal{A}$ with spectrum $X$ together with a $\mathbb{R}^{d}$-action $\alpha$ to the data of a smooth equivariant gerbe on $X$, then $f$ would be the Mackey obstruction of $[\mathcal{A}, \alpha]$. However, the construction of such a pair directly from the gerbe is difficult in general.

We now restrict ourselves to the case when $X$ is a trivial torus bundle since this case is well understood. We use the work of Echterhoff et al. [13] to compare the results of [1] with $C^{*}$-algebraic topological T-duality. We further require that $H_{0}=H_{3}=0$.

When $X=W \times \mathbb{T}^{d}$ and $f$ is not nullhomotopic, the $C^{*}$-algebraic formalism of Topological T-duality (see $[7,13]$ ) obtains a non-commutative principal $\mathbb{T}^{d}$-bundle ${ }^{8}$ as the T-dual. We argue below that when $m_{\alpha \beta}$ is not a coboundary, the smooth gerbe formalism of Belov et al. [2] determines such a bundle:

Theorem 2.2. Suppose $X=W \times \mathbb{T}^{d}$ and suppose we are given the data of a smooth $\mathbb{T}^{d}$-equivariant gerbe on $X$ in the sense of [2] with $H_{0}=H_{3}=0$. Let $\mathcal{K}$ denote the $C^{*}$-algebra of compact operators on an infinite-dimensional separable Hilbert space.

(1) The above data determines a $\mathbb{Z}^{d}$-action $\theta$ on $C_{0}(W, \mathcal{K})$, where $\mathcal{K}$ is the set of compact operators on a separable Hilbert space.

(2) If we require that $T$-duals in the $C^{*}$-algebraic formalism and the smooth equivariant gerbe formalism determine the same principal $\mathbb{T}^{d}$-bundle when $H_{1}=0$, then this $\mathbb{Z}^{d}$-action is determined uniquely.

\footnotetext{
${ }^{8}$ Note that in [13], $f$ may be nullhomotopic. Thus, their non-commutative bundles include ordinary principal torus bundles as a subset. This fact will be implicitly used below.
} 
(3) The canonical $\mathbb{Z}^{d}$-action on $C_{0}(W, \mathcal{K})$ determines a unique $C^{*}$-dynamical system $(\mathcal{A}, \alpha)$ such that $\hat{\mathcal{A}}=X$. The Dixmier-Douady invariant of $\mathcal{A}$ equals the cohomology class of $H$. The cohomology class of the Mackey obstruction of $(\mathcal{A}, \alpha)$ is equal to the class of the cocycle $m_{\alpha \beta}$ of $[2]$.

(4) The canonical $\mathbb{Z}^{d}$-action on $C_{0}(W, \mathcal{K})$ determines a unique non-commutative principal torus bundle over $W$. This bundle is isomorphic to the $C^{*}$-algebraic T-dual $\mathcal{A} \rtimes_{\alpha} \mathbb{R}^{d}$.

Proof. Since we have a trivial bundle the gerbe curvature form may be written as

$$
H=\frac{1}{2} H_{1}^{I J} d \theta_{I} \wedge d \theta_{J}+H_{2}^{I} d \theta_{I},
$$

where $H_{1}$ is a closed, integral form on $W$ and $\theta_{I}, I=1, \ldots, d$, are coordinates on $\mathbb{T}^{d}$.

(1) This is a consequence of Theorem 2.3 of [13]: The exterior equivalence classes of $\mathbb{Z}^{d}$-actions on $C_{0}(W, \mathcal{K})$ are parametrized by a group $\mathcal{E}_{\mathbb{Z}^{d}}(W)$. An element $\beta$ of this group is determined by two data:

- A Mackey obstruction map $f: W \rightarrow \mathbb{T}^{d(d-1) / 2}$.

- An isomorphism class of a principal $\mathbb{T}^{d}$ bundle $q: Y \rightarrow W$, i.e., an element of $H^{2}\left(W, \mathbb{Z}^{d}\right)$.

By Theorem 2.1 above, we already have the map $f$. The principal bundle $q$ may also be obtained from the equivariant gerbe data: By Theorem 2.2 of [2], the component $B_{1 \alpha}$ (see equation (2.1) above) of the gerbe connection $B_{\alpha}$ satisfies equation (2.17b) of [2]

$$
B_{1 \alpha}-B_{1 \beta}=d \tilde{h}_{\alpha \beta}^{I}+m_{\alpha \beta}^{I J} A_{\beta J},
$$

where $\tilde{h}_{\alpha \beta}^{I}$ are real-valued functions on $W$ satisfying the cocycle identity $^{9} \quad \tilde{h}_{\alpha \beta}^{I}+\tilde{h}_{\beta \gamma}^{I}+\tilde{h}_{\gamma \alpha}^{I}=0$. Let $g_{\alpha \beta}^{I}=\exp \left(2 \pi i \tilde{h}_{\alpha \beta}^{I}\right)$. The $g_{\alpha \beta}^{I}$ satisfy the cocycle condition

$$
g_{\alpha \beta}^{I} g_{\beta \gamma}^{I} g_{\gamma \alpha}^{I}=1
$$

on three-fold intersections $X_{\alpha \beta \gamma}$ and hence define an element ${ }^{10}$ of $H^{1}\left(W, \mathbb{T}^{d}\right)$. Hence they determine an isomorphism class of a principal $\mathbb{T}^{d}$-bundle over $W$. Now the pair $\left[f^{I J}, g^{I}{ }_{\alpha \beta}\right]$ determine a $\mathbb{Z}^{d}$-action $\theta$ on $C_{0}(W, \mathcal{K})$.

\footnotetext{
${ }^{9}$ Note that since $X$ is a trivial bundle, its transition functions $\lambda_{\alpha \beta J}, J=1, \ldots, d$, may be taken to be zero in Theorem 2.2 of [2].

${ }^{10}$ Here $\mathbb{T}^{d}$ is the sheaf of $\mathbb{T}^{d}$-valued functions on $W$ and the cohomology group is the sheaf cohomology.
} 
(2) We need to show that the choice of $\tilde{h}_{\alpha \beta}$ is canonical. After all, it is conceivable that one could construct other cocycles on $W$ which might give a completely different $\mathbb{Z}^{d}$-action on $C_{0}(W, \mathcal{K})$. However, the choice of $\tilde{h}_{\alpha \beta}$ is forced on us by the following argument: Belov et al. show that ${ }^{11}$ when $m_{\alpha \beta}$ is a trivial cocycle, the $\tilde{h}_{\alpha \beta}$ determine the isomorphism class of the T-dual principal torus bundle. We therefore require that if $f$ constructed in Theorem 2.1 is nullhomotopic, the T-dual noncommutative principal $\mathbb{T}^{d}$-bundle obtained above should reduce to a principal $\mathbb{T}^{d}$-bundle (possibly with $H$-flux) whose characteristic class should agree with that of the T-dual obtained from Belov et al. [2].

It is a well-known result that when $f=0$, i.e., $\beta$ has no Mackey obstructions, the crossed product $C_{0}(W, \mathcal{K}) \rtimes_{\beta} \mathbb{Z}^{d}$ is actually a continuous-trace algebra on an ordinary principal $\mathbb{T}^{d}$-bundle. In the $C^{*}$-algebraic formalism of Topological T-duality, the algebra $C_{0}(W, \mathcal{K}) \rtimes_{\beta} \mathbb{Z}^{d}$ is strongly Morita equivalent to the $C^{*}$-algebraic ${ }^{12} \mathrm{~T}$ dual $\mathcal{A} \rtimes_{\alpha} \mathbb{R}^{d}$ Thus, the choice of the cocycle $h$ above is canonical, as it is the only one which makes $Y$ agree with the geometric T-dual in [2].

(3) Let $\mathcal{A}=\operatorname{Ind}_{\mathbb{Z}^{d}} \mathbb{R}^{d}\left(C_{0}(W, \mathcal{K}), \theta\right)$ and $\alpha$ the induced $\mathbb{R}^{d}$-action on $\mathcal{A}$. This determines a $C^{*}$-dynamical system $(\mathcal{A}, \alpha)$ with spectrum $X$ unique up to exterior equivalence. The Dixmier-Douady class of $\mathcal{A}$ is equal to the class of $H$ in integral cohomology by construction. By the proof of Theorem 2.1 above, the homotopy class of $f$ is equal to the cohomology class $m_{\alpha \beta}$ calculated from the data of a $\mathbb{T}^{d}$-equivariant gerbe on $X$.

(4) The canonical $\mathbb{Z}^{d}$-action on $C_{0}(W, \mathcal{K})$ determines a non-commutative principal $\mathbb{T}^{d}$-bundle over $W$ namely, $C_{0}(W, \mathcal{K}) \rtimes_{\theta} \mathbb{Z}^{d}$. This is strongly Morita equivalent to the $C^{*}$-algebraic T-dual $\mathcal{A} \rtimes_{\alpha} \mathbb{R}^{d}$ by Proposition 3.4 of [5]. Since both algebras are stable, they are actually isomorphic.

Thus, we have constructed the $C^{*}$-algebraic T-dual ${ }^{13}$ from the stringtheoretic data on $X$. It is thus natural to view the non-commutative T-duals of Mathai and Rosenberg [7] as being topological approximations to the T-folds of Hull [1].

When the T-dual is geometric we may identify the correspondence space with the spectrum of $\mathcal{T} \cong \mathcal{A} \rtimes_{\left.\alpha\right|_{\mathbb{Z}^{d}}} \mathbb{Z}^{d}$. In light of the above theorem, it is natural to preserve this identification when the T-dual is non-geometric.

\footnotetext{
${ }^{11}$ Corollary 2.1 of [2].

${ }^{12}$ This is by Proposition 3.4 of Raeburn and Rosenberg [5] with $G=\mathbb{R}^{d}$ and $H=\mathbb{Z}^{d}$.

${ }^{13}$ The space that we describe as "the T-dual" above is the one obtained by applying the T-duality transformation along the all $d$ of the $S^{1}$-orbits of $X$. It is one of the possible set of T-duals of the space $X$. The T-dual that we obtain is encoded in the choice of the action $\alpha$ above.
} 
Thus, we claim that $\mathcal{T}$ is the $C^{*}$-algebra naturally associated to the T-folds of Hull. It would be interesting to construct this algebra directly from the data of the correspondence space obtained by Belov et al. [2] when the T-dual is non-geometric.

\section{D-Branes on the correspondence space}

In this section we make a few remarks on D-branes on T-folds and their connection with the formalism of Topological T-duality. We follow [11] for the theory of D-branes on Hull's T-folds.

We would like to determine the charge group of the D-branes in this background. To do this we consider the simplest case first, namely the case when there are no Mackey obstructions so both spaces are geometric. In this case, we have a commutative "diamond"

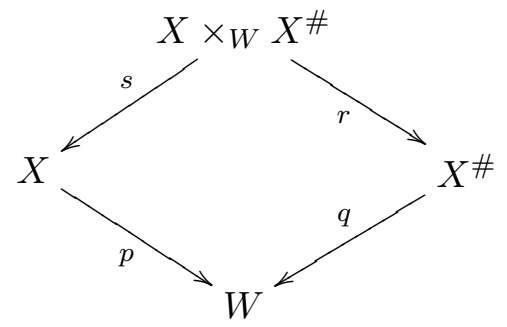

(Note that the projection operators $\Pi$ and $1-\Pi$ discussed by Hull [2] are obtained from the maps $r$ and $s$ restricted to each local coordinate patch.) From [2, Section 7], we see that in this situation, a D-brane on the correspondence space should give D-branes on $X$ and $X^{\#}$. Now due to the maps $r, s$ an element of the twisted $K$-homology of the correspondence space $K_{*}^{\tilde{H}}\left(X \times_{W} X^{\#}\right)$, will give rise to elements of $K_{*}^{H}(X)$ and $K_{*}^{H^{\#}}\left(X^{\#}\right)$, which are D-branes on $X$ and $X^{\#}$ (see for example [12]). Hence D-branes on the T-fold should be given by the elements of $K_{*}^{H}\left(X \times_{W} X^{\#}\right)$. Thus their charges should be given by the twisted $K$-theory of the correspondence space $K_{H}^{*}\left(X \times_{W} X^{\#}\right)$ which, in this case, is isomorphic to the operator-algebraic $K$-theory of $\mathcal{T}$. We conjecture that even when Mackey obstructions are present and the spectrum of $\mathcal{T}$ is not a fibered product of two spaces, this identification continues to hold. Thus, we conjecture that the charge group of D-branes on a T-fold should always be the $K$-theory of $\mathcal{T}$. We now determine the $K$-theory of $\mathcal{T}$ :

Theorem 3.1. Let $\mathcal{B}$ be a stable $C^{*}$-algebra with spectrum $W$. Let $\theta$ be a $\mathbb{Z}^{d}$-action on $\mathcal{B}$. Consider the (stable) induced algebra $\mathcal{A}=\operatorname{Ind}_{\mathbb{Z}^{d}}^{\mathbb{R}^{d}}(\mathcal{B}, \theta)$. Let the $\mathbb{R}^{d}$-action on $\mathcal{A}$ be denoted $\alpha$ and define $\mathcal{T}=\mathcal{A} \rtimes_{\alpha} \mathbb{Z}^{d}$. 
(1) We have that

$$
K_{0}(\mathcal{T}) \cong K_{1}(\mathcal{T}) \cong K_{0}(\mathcal{A})^{d} \oplus K_{1}(\mathcal{A})^{d}
$$

(2) There is a natural automorphism $\phi_{i}: K_{i}(\mathcal{T}) \rightarrow K_{i}(\mathcal{T}), i=0,1$, induced by the Connes-Thom isomorphism

\section{Proof.}

(1) We consider $\mathcal{A}$ as possessing a natural $\mathbb{Z}^{d}$ action (denoted $\alpha$ as well) obtained by restricting the $\mathbb{R}^{d}$ action to the $\mathbb{Z}^{d}$ subgroup. Consider $\mathcal{D}=\operatorname{Ind}_{\mathbb{Z}^{d}}(\mathcal{A})$. Let the $\mathbb{R}^{d}$ action on $\mathcal{D}$ be denoted $\phi$. We know that $\mathcal{D} \rtimes_{\phi} \mathbb{R}^{d}$ is strongly Morita equivalent to $\mathcal{A} \rtimes_{\alpha} \mathbb{Z}^{d}$ by Theorem 2.2 of [5]. Hence, by the Connes-Thom isomorphism theorem, it is enough to calculate $K_{*}(\mathcal{D})$. Now $\mathcal{D}$ was formed by applying the induced algebra construction to the restriction of the $\mathbb{R}^{d}$ action on $\operatorname{Ind}_{\mathbb{Z}^{d}}(\mathcal{B}, \theta)$ to $\mathbb{Z}^{d} \subseteq$ $\mathbb{R}^{d}$. Thus, the induced algebra construction collapses and $\mathcal{D} \cong \mathcal{A} \otimes$ $C\left(\mathbb{T}^{d}\right)$. Hence, by the Künneth theorem $K_{*}(\mathcal{D}) \cong K_{0}(\mathcal{A})^{d} \oplus K_{1}(\mathcal{A})^{d}$.

(2) The existence of the natural automorphism $\phi_{i}$ follows since we may repeat the above construction with $\mathcal{A} \rtimes_{\alpha} \mathbb{R}^{d}$ instead of $\mathcal{A}$, note that there is an isomorphism $\mathcal{A} \rtimes_{\alpha} \mathbb{Z}^{d} \cong\left(\mathcal{A} \rtimes_{\alpha} \mathbb{R}^{d}\right) \rtimes_{\hat{\alpha}} \mathbb{Z}^{d}$ and apply the Thom isomorphism theorem.

Note that the above proof is true for any induced algebra $\operatorname{Ind}_{\mathbb{Z}^{d}}^{\mathbb{R}^{d}}\left(C_{0}(W, \mathcal{K})\right)$. Such algebras include those with non-commutative geometries as T-duals. Thus, by our conjecture above, the charge group of D-branes on $\mathcal{T}$ will always be $K_{0}(\mathcal{A})^{d} \oplus K_{1}(\mathcal{A})^{d}$. It would be interesting to interpret this charge group physically but a more detailed ${ }^{14}$ study of D-branes on T-fold backgrounds is needed. Still, we feel that the above argument is natural enough to serve a preliminary step towards such studies.

In the above theorem we could have begun with $\mathcal{A} \rtimes_{\alpha} \mathbb{R}^{d}$ instead of $\mathcal{A}$ and then the $K$-groups would be expressed in terms of the $K$-theory of the crossed product. It would be interesting to find an expression for the $K$-theory of $\mathcal{T}$ which does not depend on the arbitrary choice of either $\mathcal{A}$ or $\mathcal{A} \rtimes_{\alpha} \mathbb{R}^{d}$.

Note that in the above theorem we could have replaced $\mathcal{A}$ with a smooth subalgebra $\mathcal{A}^{\infty} \subseteq \mathcal{A}$ and still obtained the same $K$-theory (by the Karoubi

\footnotetext{
${ }^{14}$ It is not clear, for example, whether D-branes on T-folds would carry Chan-Paton bundles on their worldvolumes.
} 
Density Theorem). In particular we could have taken the smooth subalgebra of smooth sections of the $\mathcal{K}$-bundle over $\hat{\mathcal{A}}$ which corresponds to $\mathcal{A}$. It might be interesting to try to define a smooth structure on $\mathcal{A}$ (in the sense of Connes) using the smooth structure of the gerbe on $X$. However, as currently defined, $\mathcal{A}$ is only sensitive to topological but not smooth information.

We recall that T-folds are obtained as geometrizations of parametrized families of string theories on $\mathbb{T}^{d}$. Each $\mathbb{T}^{d}$ fiber is the background for an exactly solvable string theory which could have D-brane excitations. Now consider families of these D-branes one in each fiber parametrized by cycles in the base $W$. These would be obvious candidates for D-branes on T-folds. Such D-branes would wrap lifts of cycles on the base $W$ to the correspondence space and also wrap the torus fiber. We note that in [11] such configurations have been studied from the string-theoretic point of view in backgrounds which are geometrizations of T-folds, i.e., in the correspondence space $\mathcal{C}$ of [2]. We would like to determine the analog of these types of D-brane configurations in the formalism of Topological T-Duality.

It is well known that the charge group of D-branes on a space $X$ is the topological $K$-theory of $X$. The D-brane configuration described above would correspond to sections of a bundle of $K$-theory groups over $W$ in the sense of [13].

The authors of [13] study $C^{*}$-bundles: Roughly speaking, these are $C^{*}$-algebras whose elements are sections of bundles ${ }^{15}$ of $C^{*}$-algebras over a base space $W$. For example, the algebras $\mathcal{A}, \mathcal{T}$ and $\mathcal{A} \rtimes_{\alpha} \mathbb{R}^{d}$, discussed above, are all $C^{*}$-bundles over $W$.

To a $C^{*}$-bundle $\mathcal{A}(W)$ over a topological space $W$ satisfying certain conditions (see [14] for details), Echterhoff et al. [13] associates a bundle of abelian groups: the fiber of this bundle over a point $x \in W$ is the $K$-theory of the fiber of $\mathcal{A}(W)$ at that point. They also associate to this bundle a monodromy map: If $\mathcal{A}_{w}$ is the fiber of $\mathcal{A}(W)$ over $w \in W$, they define a map $\pi_{1}(W) \rightarrow \operatorname{Aut}\left(K_{*}\left(A_{w}\right)\right)$. In addition the authors of [13] show that the $K$-theory group of $\mathcal{A}(W)$ (for example, the charges of D-branes on $\mathcal{T}$ ) may be obtained from a spectral sequence whose $E^{2}$-term is a certain family of groups calculated using sections of the $K$-theory bundle of $\mathcal{A}(W)$. This connection between parametrized families of D-branes and the $K$-theory of $\mathcal{T}$ (which is the charge group of D-branes) is interesting.

Do such $K$-theory bundles exist for the $C^{*}$-algebra $\mathcal{T}$ of the correspondence space? If so, continuous sections of the $K$-theory bundle of $\mathcal{T}$ would

\footnotetext{
${ }^{15}$ These bundles do not need to be locally trivial.
} 
correspond to parametrized families of D-branes on the T-fold associated to $\mathcal{T}$. The following result holds for induced $C^{*}$-algebras $\mathcal{A}$ :

Theorem 3.2. Let $\mathcal{B}=C_{0}(W, \mathcal{K})$ and let $\theta$ be a $\mathbb{Z}^{d}$-action on $\mathcal{B}$. Consider the induced algebra $\mathcal{A}=\operatorname{Ind}_{\mathbb{Z}^{d}} \mathbb{R}^{d}(\mathcal{B}, \theta)$. Let the $\mathbb{R}^{d}$-action on $\mathcal{A}$ be denoted $\alpha$ and define $\mathcal{T}=\mathcal{A} \rtimes_{\alpha} \mathbb{Z}^{d}$.

(1) $\mathcal{T}$ is a $C^{*}$ fibration over $W$ with fibers $\mathcal{F} \cong C\left(\mathbb{T}^{d}, \mathcal{K}\right) \rtimes_{\left.\alpha\right|_{\mathbb{Z}^{d}}} \mathbb{Z}^{d}$.

(2) It is a $K$ fibration in the sense of Definition 4.1 of [13] and hence possesses a K-theory bundle and a monodromy map $\rho: \pi_{1}(W) \rightarrow$ $\operatorname{Aut}\left(K_{*}(\mathcal{F})\right)$.

(3) When there is a geometric T-dual, the image of the monodromy map lies in $\mathrm{GL}(2 d, \mathbb{Z})$.

Proof.

(1) It is clear that $\mathcal{T}$ is a $C_{0}(W)$-algebra: recall the crossed product of a $C_{0}(W)$-algebra by a spectrum fixing action of a group is also a $C_{0}(W)$ algebra. Now, $\mathcal{T} \cong \mathcal{A}^{\#} \rtimes_{\left.\alpha \#\right|_{\mathbb{Z}^{d}}} \mathbb{Z}^{d}$ but $\mathcal{A}^{\#}$ which is, by definition, $A \rtimes_{\alpha} \mathbb{R}^{d}$ is isomorphic to $C_{0}(W, \mathcal{K}) \rtimes_{\theta} \mathbb{Z}^{d}$ (since $\mathcal{T}$ is stable). Thus, $\mathcal{T}$ is a $C^{*}$ fibration over $W$. Now $\mathcal{T}$ is by definition $\mathcal{A} \rtimes_{\alpha} \mathbb{Z}^{d}$. $\mathcal{A}$ is a $C^{*}$-bundle over $W$ with fibers $C\left(\mathbb{T}^{d}, \mathcal{K}\right)$. Since $\left.\alpha\right|_{\mathbb{Z}^{d}}$ is spectrum fixing, the fibers of the fibration $\mathcal{T}$ are $\mathcal{F} \cong C\left(\mathbb{T}^{d}, \mathcal{K}\right) \rtimes_{\left.\alpha\right|_{\mathbb{Z}^{d}}} \mathbb{Z}^{d}$.

(2) By Lemma 8.4 of [13], $\mathcal{A}$ is a $K$ fibration. Since $\mathcal{T}$ is the crossed product of $\mathcal{A}$ by a spectrum-fixing $\mathbb{Z}^{d}$-action, by Remark 2.3 Part (1) of [14], $\mathcal{T}$ is a $K$ fibration as well. The existence of a $K$-theory bundle and a monodromy map follow from Proposition 4.2 of [13].

(3) In the geometric case (i.e., when the Mackey obstruction vanishes), the fibers of $\mathcal{T}$ over the base are just $C\left(\mathbb{T}^{2 d}, \mathcal{K}\right)$. Since $K_{i}\left(C\left(\mathbb{T}^{2 d}, \mathcal{K}\right)\right) \cong$ $\mathbb{Z}^{2 d}, i=0,1$, the image of the monodromy map is $\operatorname{Aut}\left(\mathbb{Z}^{2 d}\right) \cong \mathrm{GL}(2 d, \mathbb{Z})$.

Since the $K$-theory groups of each fiber are discrete groups, if the $K$-theory bundle is trivial, the sections would be constant. This would correspond to choosing a D-brane configuration on $W$ which restricts to the same D-brane on each fiber. In general, if the bundle possesses a monodromy, there could be non-constant sections. For example, we could pick a D0-brane in every fiber and a T-dual D1-brane on applying the monodromy. Since the brane worldvolume jumps discontinuously, such D-brane configurations cannot exist when the background is geometric. However, they might exist in non-geometric backgrounds. In fact, these configurations have been 
observed in several physical examples in [11] where they are termed "generalized" D-branes. In [11], parametrized families of D-branes for which this does not occur are termed "geometric" D-branes. The authors also consider generalized D-branes which "return to themselves" only after (finitely) many traversals of such loops.

What would be the analog of these in Topological T-duality? We suggest the following construction: As shown in Proposition 4.2 of Ref. [13] there is an action of $\pi_{1}(W)$ on the $K$-theory bundle of the algebra $\mathcal{T}$. We can define geometric families of D-branes in the $C^{*}$-algebraic formalism as constant sections of this bundle. Non-geometric families would then correspond to non-constant sections. Recall that the worldvolume of a generalized D-brane "returns to itself" after a finite number of circuits of the cycle it wraps. This implies that the monodromy of the associated section of the $K$-theory bundle should be cyclic. That is, in the formalism of Topological T-duality generalized D-branes ${ }^{16}$ would exist on $\mathcal{T}$ if at some point $w$ in $W$, the image of the monodromy map $\rho: \pi_{1}(W) \rightarrow \operatorname{Aut}\left(K_{*}\left(\mathcal{A}_{w}\right)\right)$ was a finite cyclic subgroup of $\operatorname{Aut}\left(K_{*}\left(\mathcal{A}_{w}\right)\right)$. Obviously, when the monodromy map is trivial, all D-brane configurations are geometric.

\section{Example: $\mathbb{T}^{3}$ with non T-dualizable $H$-flux}

In this section we study in detail the T-fold associated to a spacetime which is a trivial $\mathbb{T}^{2}$-bundle over $\mathbb{T}$. First, we make some remarks on the T-fold formalism applied to spacetimes which are trivial torus bundles over $W$.

Consider a space of the form $\mathbb{T}^{d} \times W$. Let $y_{i}, i=1, \ldots, n$, be local coordinates on $W$ and $\theta_{k}, k=1, \ldots, d$, be coordinates on the $\mathbb{T}^{d}$ fiber. Assume that the metric on the space is the product metric of the flat metric on $\mathbb{T}^{d}$ with the metric on $W$. The $B$ field may then be written $B_{0 \alpha}^{I J} d \theta_{I} \wedge d \theta_{j}+\cdots$. Here, the dots denote terms in the $B$ field containing one or zero $d \theta_{I}$ 's. It is clear that only $B_{0}$ will enter the expression for $\mathcal{H}$ because only the $B_{0}$ component of the $B$ field is supported completely on the torus fiber. The other components have non-trivial support along the base $W$. Thus, the restriction of the $B$ field to the $\mathbb{T}^{d}$ fiber is locally $B_{0 \alpha}^{I J}$. If we examine the form for $\mathcal{H}$ we see that, in the T-fold metric, the $B_{0 \alpha}^{I J}$ appear along antidiagonal blocks. As noted in Theorem 2.2 of [2], we have

$$
B_{0 \alpha}^{I J}=B_{0 \beta}^{I J}-m_{\alpha \beta}^{I J} .
$$

\footnotetext{
${ }^{16}$ In the sense of [11].
} 
Hence, a non-zero class $m_{\alpha \beta}$ causes the metric $\mathcal{H}$ to have non-trivial offdiagonal components. We claim that it is the twisting of the T-fold by these components of $\mathcal{H}$ that creates the monodromy in the total space of the T-fold (as noted in [2]).

Pick a open cover of $\mathbb{T}$ consisting of two open sets, with $U_{1}$ being the complement of 0 and $U_{2}$ the complement of its antipodal point. Let $x$ be the coordinate on the base $\mathbb{T}$ and $y, z$ be the coordinates on the $\mathbb{T}^{2}$ fiber. We pick the flat metric on $\mathbb{T}^{3}$ and an $H$-flux $H=N d x \wedge d y \wedge d z$. We pick the $B$ field $B=N x d y \wedge d z$ corresponding to this $H$-flux.

We recall that the connection forms on $\mathbb{T}^{3}$ are the one-forms $d y, d z$. Therefore, comparing with equation (2.1) above, we see that $B_{0 \alpha}=N x$. It is then clear that on changing charts, we have that $B_{01}=B_{02}-N$. Now $m_{\alpha \beta}$ is an integral two-cocycle on $W$ and as such, is determined by specifying integers for each pairwise intersection $U_{\alpha \beta}$. With the given choice of charts, the cocycle $m_{\alpha \beta}$ has value $N$ on $U_{1} \cap U_{2}$. Note that changing the coordinate functions on $U_{\alpha}$ will simply give a cohomologous cocycle. In addition, using Theorem 2.1 in Section 2 above we see that the Mackey obstruction map is exactly the map $x \rightarrow \exp (2 \pi i N x)$. This is the Mackey obstruction map constructed for an induced algebra with spectrum $\mathbb{T}^{3}$ by Mathai and Rosenberg in Proposition 4.1 of [7]. Note that the construction of [7] exists for any continuous Mackey obstruction function. However, we will always obtain a smooth function as a Mackey obstruction. The reason, as mentioned in the introduction to Section 2, is that Topological T-duality is a topological approximation (a continuous-trace algebra with a $\mathbb{R}^{d}$ action) of a smooth $\mathbb{T}^{d}$ equivariant gerbe with connection.

Following [11, Section 2.3], let

$$
\left(\begin{array}{c}
X^{i} \\
\tilde{X}^{i}
\end{array}\right)=\left(\begin{array}{c}
y \\
z \\
\tilde{y} \\
\tilde{z}
\end{array}\right)
$$

be the coordinates on the corresponding T-fold. The metric $\mathcal{H}$ on the T-fold is ${ }^{17}$

$$
\mathcal{H}=\left(\begin{array}{cccc}
1+(N x)^{2} & 0 & 0 & N x \\
0 & 1+(N x)^{2} & -N x & 0 \\
0 & -N x & 1 & 0 \\
N x & 0 & 0 & 1
\end{array}\right)
$$

\footnotetext{
${ }^{17}$ See equation $(2.20)$ of $[11]$.
} 
(Note that the metric is well defined even though the double T-dual is not a geometric space. Note also that the metric and $B$ field on the double T-dual may be recovered from $\mathcal{H}$ as discussed in the introduction.)

A detailed analysis of D-branes on T-fold backgrounds has been done in [11, Section 3.4]. A D-brane on such a background is specified by a projection valued function $\Pi_{D}$ on $W$ (a "Dirichlet projector") which picks out the directions with Dirichlet boundary conditions on each $\mathbb{T}^{2 d}$ fiber over $W$. This also defines a Neumann projector $\Pi_{N}=\left(\mathbf{1}-\Pi_{D}\right)$ on the T-fold which determines the Neumann directions on each fiber. The D-brane boundary conditions are determined by $\Pi_{D} \partial_{0} \mathbb{X}=0$. We can see that these determine two sets of boundary conditions $\Pi \Pi_{D} \partial_{0} \mathbb{X}=0$ and $\tilde{\Pi} \Pi_{D} \partial_{0} \mathbb{X}=0$. Recall that the $\Pi \mathbb{X}$ were coordinates on $X$ and the $\tilde{\Pi} \mathbb{X}$ were coordinates on the T-dual $X^{\#}$. It is clear that the two sets of boundary conditions above determine parametrized families of D-branes on $X$ and $X^{\#}$, respectively. These may not actually be D-branes as the boundary conditions may possess a non-trivial monodromy. However, one can check that whenever a D-brane wrapping the T-fold of $\mathbb{T}^{3}$-with $H$-flux is an allowed D-brane, the projections of this brane to $X$ and $X^{\#}$ are also allowed D-branes.

We may use this phenomenon to justify the expression for $K_{i}(\mathcal{T})$ that we have obtained in Theorem 3.1 above, at least for $\mathbb{T}^{2}$ fibrations. Suppose $\mathcal{A}$ satisfies the hypotheses of Theorem 3.1 above, then the spectrum of $\mathcal{A}$ is a trivial $\mathbb{T}^{2}$-bundle over $W$, and the $K$-theory of the associated T-fold is

$$
K_{i}(\mathcal{T}) \cong\left(K_{0}(\mathcal{A})\right)^{2} \oplus\left(K_{1}(\mathcal{A})\right)^{2} .
$$

Now, using the Thom isomorphism, this may be written as

$$
K_{i}(\mathcal{T}) \cong K_{0}(\mathcal{A}) \oplus K_{0}\left(\mathcal{A} \rtimes_{\alpha} \mathbb{R}^{2}\right) \oplus K_{1}(\mathcal{A}) \oplus K_{1}\left(\mathcal{A} \rtimes_{\alpha} \mathbb{R}^{2}\right)
$$

We could interpret this as saying that a D-brane on $\mathcal{T}$ would determine D-branes on $\mathcal{A}$ and $\mathcal{A} \rtimes_{\alpha} \mathbb{R}^{2}$ and further that the dimension of these D-branes need not have the same parity as that of the D-brane on $\mathcal{T}$. A closer study of D-branes on $\mathcal{T}$ might give insight into the formula for $K_{i}(\mathcal{T})$ in the case $d \geq 2$.

\section{Conclusion}

In this paper we have argued that the T-folds in string theory are naturally related to the non-commutative T-duals of [7]: In a particular class of examples, the non-commutative T-duals of Ref. [7] may be naturally obtained 
from string theoretic data. It would be interesting to extend Theorem 2.2 to the case when $H_{3} \neq 0$. One would have to study exterior equivalence classes of $\mathbb{Z}^{d}$-actions on continuous trace algebras on $W$ with non-zero DixmierDouady invariant. It would also be interesting to extend the above theorem to non-trivial principal torus bundles.

We suspect that it should be possible to obtain the $C^{*}$-algebra $\mathcal{T}$ from the data defining the non-principal $\mathbb{T}^{2 d}$-bundle $\mathcal{C}$ which is the correspondence space of $[2]$.

We have also argued that D-branes on T-folds have an analogue in the $C^{*}$-algebraic formalism. It would be interesting to pursue these analogies further.

\section{Acknowledgments}

We acknowledge financial support from the Australian Research Council through the Discovery Project "Generalized Geometries and their Applications".

\section{References}

[1] C. M. Hull, A geometry for non-geometric string backgrounds, J. High Energy Phys. 10 (2005), 065; arXiv:hep-th/0406102.

[2] D. M. Belov, C.M. Hull and R. Minasian, T-duality, gerbes and loop spaces, arXiv:0710.5151.

[3] S. Hellerman, J. McGreevy and B. Williams, Geometric construction of nongeometric String Theories, J. High Energy Phys. 01 (2004) 024; arXiv: hep-th/0208174.

[4] D. Vegh and J. McGreevy, Semi-flatland, J. High Energy Phys. 10 (2008) 068, arXiv:0808.1569.

[5] I. Raeburn and J. Rosenberg, Crossed products of continuous-trace $C^{*}$-algebras by smooth actions, Trans. Am. Math. Soc. 305 (1) (1988), 1-45.

[6] V. Mathai and J. Rosenberg, T-duality for torus bundles with H-fluxes via noncommutative topology, Commun. Math. Phys. 253 (2005), 705-721; arXiv:hep-th/0401168.

[7] V. Mathai and J. Rosenberg, T-duality for torus bundles with H-fluxes via noncommutative topology II, Adv. Theor. Math. Phys. 10 (2006), 123-158; arXiv:hep-th/0508084. 
[8] P. Bouwknegt, J. Evslin and V. Mathai, On the topology and H-flux of T-dual manifolds, Phys. Rev. Lett. 92 (2004), 181601; arXiv:hep-th/0312052v2.

[9] P. Grange and S. Schäfer-Nameki, T-duality with H-flux: noncommutativity, T-folds and $G \times G$-structures, Nucl. Phys. B770 (2007), 123-144, arXiv:hep-th/0609084.

[10] P. Bouwknegt, K. Hannabuss and V. Mathai, T-duality for principal torus bundles and dimensionally reduced Gysin sequences, Adv. Theor. Math. Phys. 9 (2005), 749-773, arXiv: hep-th/0412268.

[11] A. Lawrence, M. B. Schulz and B. Wecht, D-branes in nongeometric backgrounds, J. High Energy Phys. 07 (2006), 038, arXiv:hep-th/0602025.

[12] R. M. G. Reis and R. J. Szabo, Geometric K-homology of flat D-branes, Commun. Math. Phys. 266 (2006), 71-122; arXiv:hep-th/0507043.

[13] S. Echterhoff, R. Nest and H. Oyono-Oyono, Principal noncommutative torus bundles, preprint, available at

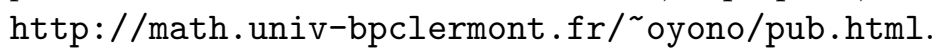

[14] S. Echterhoff, R. Nest and H. Oyono-Oyono, Fibrations with noncommutative fibers, preprint, available at http:// math. univ-bpclermont.fr/ ${ }^{\sim}$ oyono/pub.html. 
